\title{
Multi-liquid precise measurement control system based on UKF
}

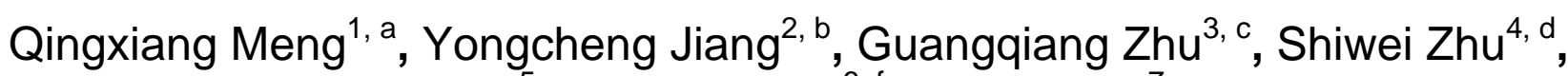 \\ Meaza $^{5, \mathrm{e}}$, Xiuping Hua ${ }^{6, \mathrm{f}}$, Qinglin $\mathrm{Han}^{7, \mathrm{~g}}$ \\ ${ }^{1}$ College of Mechanical Engineering, Jiamusi University, Jiamusi 154007, China; \\ ${ }^{2}$ College of Mechanical Engineering, Jiamusi University, Jiamusi 154007, China. \\ amengqiangxiang714@126.com, bjiangyongcheng@126.com, ' $13604863375 @ 139 . c o m$
}

Keywords: programmable logic controller, traceless Kalman filter, liquid mixture batching system.

\begin{abstract}
At present, the lots of ongoing mixture and batching machine unit abroad exist unsure interact dynamic influence in production and operation flow process, facility dynamic physical characteristic, computation precision difference, and many factors difficult to analysis. there are multiple factors that makes it difficult to realize material dynamic precise computation in batching process. Now the problem of computation precise has already become one of the general questions in mixture and batching unit facility, therefore, this article put forward a kind of control method. Which is called UKF that adopting or dispose computation acquisition signal elicited by PLC smoothing for improving the stability of the signal. As indicated in the experiment result when comparing with traditional control system, this is new type of control system design scheme which enhance effectively the stability of signal which have comprehensive implement prospect in mechanical control system.
\end{abstract}

\section{Introduction}

In the case of material machining, due to the complexity of machine structure the high speed of machine flow switch, which always there is a need of mixing different types of liquid in a suitable time and condition. Which makes the computation data exist dynamic wave and uncertainty, aggravating of the mixture helps for the developing precise computation system, in the case of material machining. Developing the process computation system can fully master or specializes the dynamic characteristic computation data from the hardware to the software level computation system for designing the program. From computation system hardware level to software control program design, data display statistics, mechanical structure optimize and scene production installing and debugging and many aspects proceed deeply analysis.

In the actual production, to insure the stability of production process and the precise ratio of material, needs high precise proceed and certain successive certain computation form types of material or some of them. it computation is precise or not and the precision of computation is stable or not which doubtless is rhe key of if the liquid process field craft system normal operation ${ }^{[1]}$.adopting precise, reliable auto-batch system is important to enhance the stability of facility and insure the quality of production.

This article outwards a type of automates parameter controller based on the smoothing of UKF, the program adopt and dispose the acquisition weight signal, significantly decrease environment noise and disturb influence ${ }^{[2]}$, and enhance the stability and dynamic of system.

\section{A Control System Based on UKF filter}

In 1995, Julier introduced the sample of UKF algorithm method for approximate distribution of nonlinear, avoid computing system equation of Jacobian matrix, the filtering precision is improved greatly. UKF algorithm is mainly based on the unscented transformation (UT transform), using UT transform after filtering state variables to estimate, can achieve the result of improved nonlinear filtering and improve the positioning accuracy. At present, the UKF algorithm has been widely used in filtering operation control system ${ }^{[3]}$. 
For discrete nonlinear systems

1) The initialization

2) Computing sigma points

$$
\begin{gathered}
\hat{x}_{0}=E\left[x_{0}\right] \\
P_{0}=E\left[\left(x_{0}-\hat{x}_{0}\right)\left(x_{0}-\hat{x}_{0}\right)^{T}\right] \\
k \in\{1, \cdots, \infty\}
\end{gathered}
$$

$$
\left\lfloor\hat{x}_{k-1} \hat{x}_{k-1}+\sqrt{\left(d_{x}+\lambda\right) P_{k-1}} \hat{x}_{k-1}-\sqrt{\left(d_{x}+\lambda\right) P_{k-1}}\right\rfloor
$$

3) To predict

$$
\begin{gathered}
\chi_{k \mid k-1}^{*}=F\left[\chi_{k-1}, u_{k-1}\right] \\
\bar{x}_{k}=\sum_{i=0}^{2 d_{x}} W_{i}^{(m)} \chi_{i, k \mid k-1}^{*} \\
P_{\bar{k}}=\sum_{i=0}^{2 d_{x}} W_{i}^{(c)}\left[\chi_{i, k \mid k-1}^{*}-\tilde{x}_{k}\right] \cdot\left[\chi_{i, k \mid k-1}^{*}-\tilde{x}_{k}\right]^{T}+R^{v} \\
\chi_{k \mid k-1}=\left[\tilde{x}_{k} \tilde{x}_{k}+\sqrt{\left(d_{x}+\lambda\right) P_{\bar{k}}} \hat{x}_{\bar{k}}-\sqrt{\left(d_{x}+\lambda\right) P_{\bar{k}}}\right] \\
Y_{k \mid k-1}=H\left\lfloor\chi_{k \mid k-1}\right\rfloor \\
\bar{y}_{k}=\sum_{i=0}^{2 d_{x}} W_{i}^{(m)} Y_{i, k \mid k-1}
\end{gathered}
$$

As shown in figure 1 is mix ingredients based on UKF filter system schematic diagram

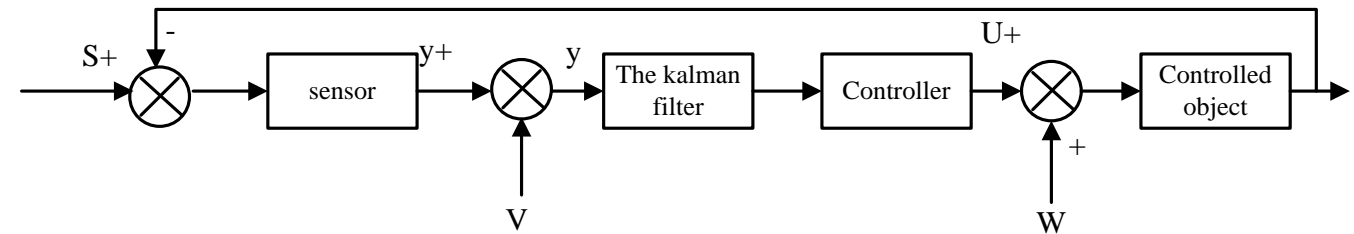

Figure 1 mixed batching control system based on UKF

\section{The Simulation Effect}

The main purpose of the experiment is for obtaining stable data weight. During the experiment, we have been gotten the actual state variable by using sensor weight ${ }^{[4]}$. Therefore, we use the kalman filtering model for one-dimensional scalar, model. The following is the process of the experiment. Which shows the collected sensor data that obtained from the $\{21,22,23\}$ using UKF filter method from PLC to signal filtering process, as shown in figure 2.

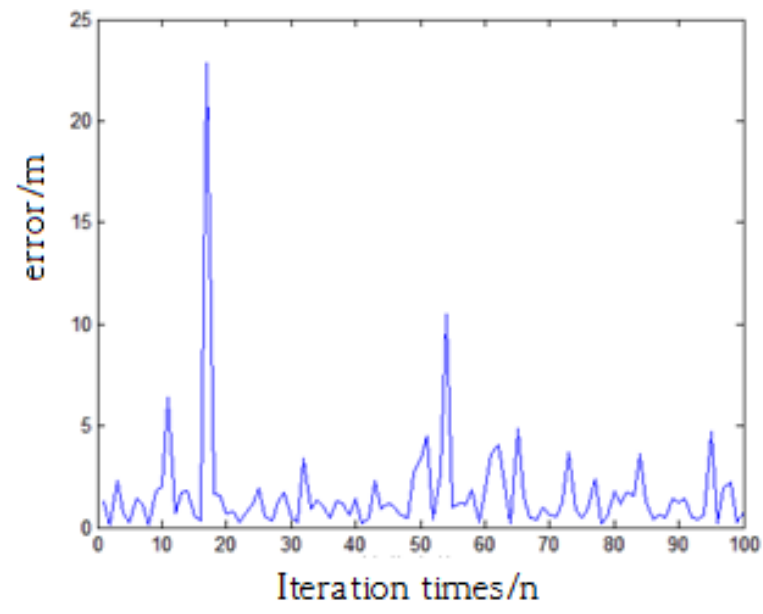

Figure 2 UKF filter effect

As can be seen from the figure 2, after the acquisition of PLC, the UKF filter, curve error is less and has poor ability. Therefore, the UKF filter PLC for signal filtering system. 


\section{The Practical Application}

In Heilongjiang world cable, a subsidiary of embellish technology co., Ltd. (Heilongjiang Orient Science \& Technology co., Ltd) for field application.

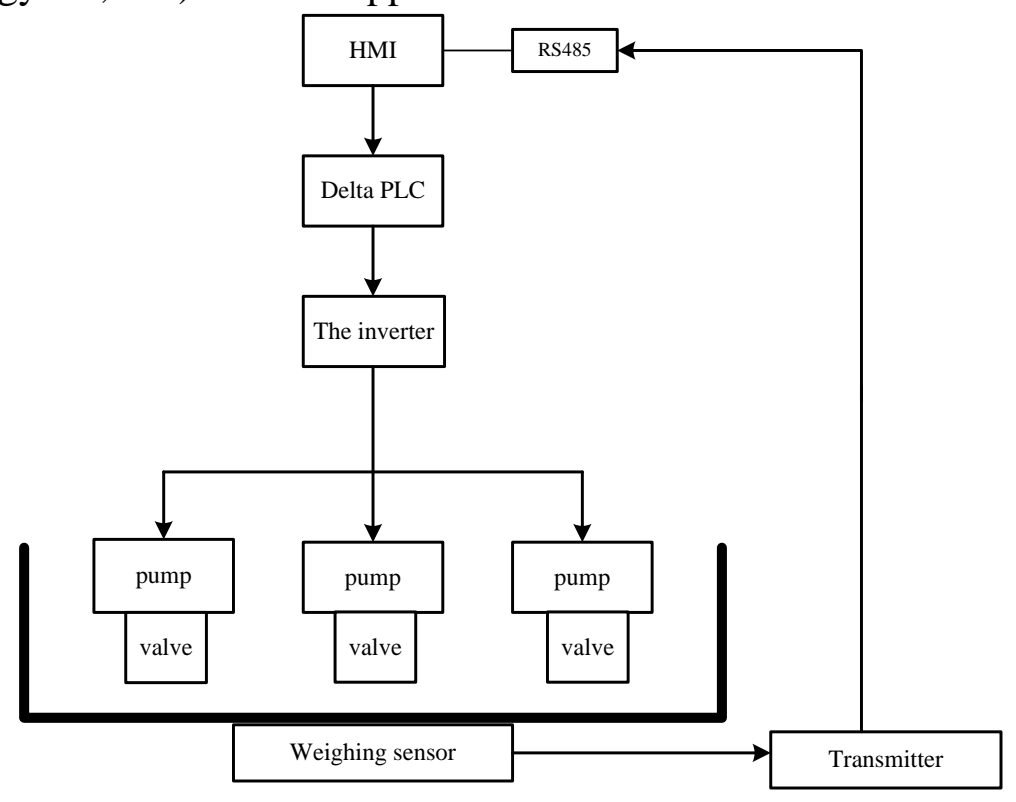

Figure 3 process diagram

According to the process shown in diagram 3, the object of the research us to obtain the weight of liquid material using sensor. Based on judgments of experience and science, in the same place value is a constant. Assumes that the nonlinear system $y=f(x)$, in which the dimension of $x$ for $n$ [5], average value were $\bar{x}$, variance were $P_{x}$, in order to estimate $y$, to construct $(2 n+1)$ dimensional vector $\chi$ as follows:

$$
\chi=\left[\chi_{0}, \chi_{1}, \cdots, \chi_{L}, \chi_{L+1}, \cdots, \chi_{2 n}\right]
$$

Which $\chi_{i}(i=0,1, \cdots, 2 n)$ is called a vector $\sigma$, its specific form is:

$$
\left\{\begin{array}{c}
\chi_{0}=\bar{x} \\
\chi_{i}=\bar{x}+\sqrt{(n+\lambda)}\left\{\operatorname{chol}\left(P_{x}\right)\right\}_{i}^{T} \quad i=1, \cdots, n \\
\chi_{i}=\bar{x}-\sqrt{(n+\lambda)}\left\{\operatorname{chol}\left(P_{x}\right)\right\}_{i}^{T} \quad i=n+1, \cdots, 2 n
\end{array}\right.
$$

Type, $\operatorname{chol}\left(P_{x}\right)$ represents Cholesky decomposition of $P_{x}$, and $\left\{\operatorname{chol}\left(P_{x}\right)\right\}_{i}^{T}$ represents the Cholesky decomposition of transposed of $P_{x}$ (that is, the lower triangular matrix) of the $i$ column, $\lambda$ defined as

$$
\lambda=\alpha^{2}(n+k)-n
$$

Type, $\alpha$ to express $\sigma$ the degree of vector distribution in the $\bar{x}$ near its scope, Its value range is $10^{-4} \leq \alpha \leq 1, \kappa$ usually set to zero value.

Will $\chi$ each column ( $\sigma$ vector)

Resulting from the nonlinear function $y=f(x)$ transformation:

$$
y_{i}=f\left(\chi_{i}\right) i=0,1, \cdots, 2 n
$$

Thus the mean value $y=\left[y_{0}, y_{1}, \cdots, y_{2 n}\right]$ was $\bar{y}$ and variance $P_{y}$, respectively:

$$
\begin{gathered}
\bar{y}=\sum_{i=0}^{2 n}\left(W_{i}^{(m)} y_{i}\right) \\
P_{y}=\sum_{i=0}^{2 n}\left[W_{i}^{(c)}\left(y_{i}-\bar{y}\right)\left(y_{i}-\bar{y}\right)^{T}\right]
\end{gathered}
$$

The state equation is: 


$$
x_{k}=x_{k-1}+w_{k}
$$

The Measurement equation is

$$
z_{k}=x_{k}+v_{k}
$$

Type: ${ }^{X_{k}}$ as state estimation variables; ${ }^{W_{k}}$ as process noise variables, here goes $10^{-4} ; v_{k}$ as the observation noise variables, value of 0.1 here ${ }^{[6]}$.

Liquid mixing ingredients control system based on UKF which has be put into application in the factory, improve the productivity, reduce the cost and obtain stable signals. The control effect is very significant, the control accuracy is greatly improved, and the operation is stable. In this article, as show in figure4, sample of actual production data mentioned at different times. Finally it is concluded that during the process of production, there is complicated fluctuation in the case of measuring the curve for each material analysis and for typical. Therefore, the UKF filter can solve the problem of signal wave for data analysis. (Orange curve after filtering, blue for filtering before curve).

Weight/acquisition

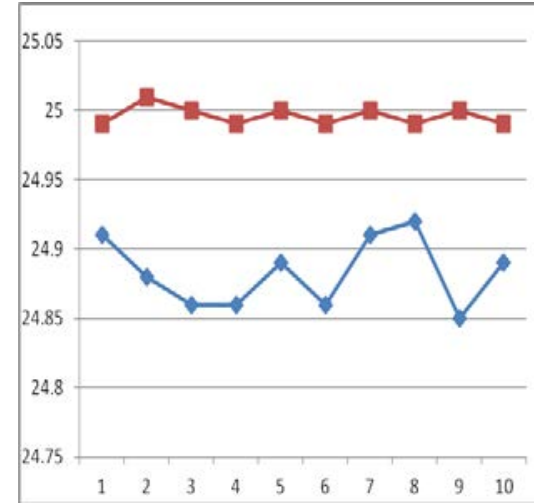

Figure 4 filter before and after contrast

\section{Summary}

As in the above research shown that signal measuring through UT transform is a good and it is the best method that helps to predict the new state and observation system and the experiment verified that the UKF system can accurately projections for nonlinear variable state that estimates for the Gaussian noise accuracy up to the third nonlinear system to show its effect remarkable and strong. Because the good performance of the UKF, the filter process does not need partial derivative the matrix calculation for the nonlinear system. Through the actual operation, the robust system performance has good, precision control system.

\section{Acknowledgments:}

Jiamusi University Master of Science and technology innovation project (LZZ2014_007), (LZZ2015_002).

Jiamusi University science and technology innovation team (Cxtd-2013-01).

Scientific Research Foundation for the Returned Overseas Chinese Scholars, State Education Ministry (20141685).

\section{References}

[1] YanxingGuang, sun. PLC application in the liquid mixing device [J]. Mechanical and electrical integration, 2004, (3): P63-65.

[2] Shirui Chen, xiangqun Wang. The high accuracy in the filling production line automation technology application [J]. Journal of packaging and food machinery, 2004.

[3] Hou Rui. Particle filter and U - the application of particle filter in CDMA multiuser detection [D]. 
Beijing Jiaotong University, 2008.

[4] Mauro D M,Simultaneous localization and map building for A team of cooperating robots :A set membership approach[J].IEEE transactions on robotics\&automation,2003,19(2):P238-248.

[5] Automatic Control, 2000, 45(3):P477-482

[6] S.J.Julier,J.K.Uhlmann. Unscented filtering and nonlinear estimation[C]. Proceedings of the IEEE, 2004, 92(3)

P401-422. 